\title{
Chemical and textural analysis of rocks with the scanning electron microscope
}

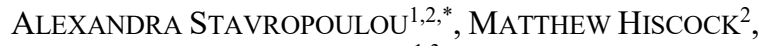 \\ BALZ SAMUEL KAMBER ${ }^{1,3}$ AND JUAN-DIEGO \\ RODRIGUEZ-BLANCO ${ }^{1}$ \\ ${ }^{1}$ Department of Geology, Trinity College Dublin, The \\ University of Dublin, Dublin 2, D02 PN40, Ireland \\ ${ }^{2}$ Oxford Instruments Nanoanalysis, Halifax Rd, High \\ Wycombe, HP12 3SE, UK \\ ${ }^{3}$ School of Earth, Environmental and Biological Sciences, \\ Queensland University of Technology, Brisbane City, \\ QLD 4000, Australia \\ *stavropa@tcd.ie
}

The demand for resources is pressing as ever: Exploration programs target high-grade resources, but these are becoming harder to find. As such, there is a constant drive to improve efficiency in the mining industry. This study focusses on how maximising the amount of information that can be extracted from a thin section sample of a rock can improve extraction efficiency.

The main advantage of analysing rock thin sections instead of powdered samples, as is the case in several traditional techniques (e.g. powder X-ray diffraction, X-ray fluorescence, atomic absorption spectroscopy, inductively coupled plasma - optical emission spectrometry etc.), is that rock texture is preserved. This means that $\mathrm{X}$-ray techniques, such as energy dispersive X-ray spectroscopy (EDS) and electron backscatter diffraction (EBSD), can be employed for in-situ, bulk rock analysis. A huge amount of information can be gathered from a single sample, incorporating both chemical and structural information, from the same location, allowing direct correlation of compositional and structural properties. The implications are of great importance, as the mining industry is notorious for the vast amounts of energy it requires to operate (e.g., comminution), with some mineral processing procedures being particularly demanding and largely inefficient (e.g, overgrinding). Here, an example of multi-technique analysis in the SEM is presented, illustrating the information that can be extracted from a number of relevant, representative samples.

By enhancing the amount of valuable data acquired in a single analysis session, it is possible to acquire all the necessary information to both improve the efficiency of minerals processing and reduce environmental impact by conserving energy, and thus reducing carbon dioxide emissions. 